\title{
Prevención de riesgos laborales en la maricultura artesanal de Ecuador.
}

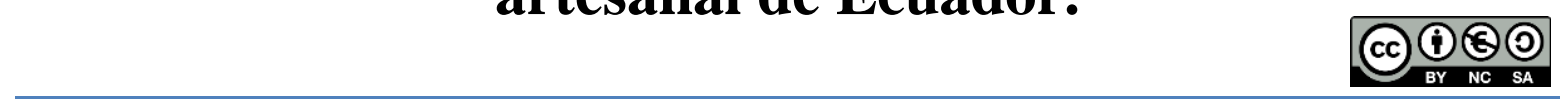

Prevención de riesgos laborales en la maricultura artesanal de Ecuador.

Milton Gabriel Montúfar Romero ${ }^{1}$, Ulises Tiberio Avendaño Villamar. ${ }^{2}$ \& Walter Edgar Ruiz Chóez. ${ }^{3}$ Xavier Napoleón Icaza Torres. ${ }^{4} \&$ Alex Javier Alta Tierra. ${ }^{5}$

Recibido: 09-12-2017 / Revisado: 14-02-2018 Aceptado: 11-03-2018/ Publicado: 01-04-2018

\begin{abstract}
.
DOI: https://doi.org/10.33262/cienciadigital.v2i2.106

Mariculture is a developing sector that is associated with various risks of working at sea. There are not exhaustive studies of these dangers in Ecuador, and there is great uncertainty about their scope. The question proposed for this investigation was: What is known about occupational risks for artisanal seafood farmers? Through open interviews and visits made to all Artisanal Fishermen Cooperatives engaged in this activity, some occupational hazards were identified and assessed using the Occupational Safety Risk Index. In this analysis, causes of death include drowning, and fatal brain injury; the causes of disability are associated with back injuries; the causes of serious injuries include falls on the boat, risks associated with diving, projections of particles to the eyes, cuts or abrasion on the skin, and skin diseases caused by prolonged exposure to solar radiation without adequate protection; and in the causes of moderate injuries were determined dermatological conditions caused by direct contact with aquatic organisms.
\end{abstract}

Keywords:. Mariculture, Prevention, Risk, Index, Occupational.

\footnotetext{
${ }^{1}$ Instituto Nacional de Pesca, Ecuador, ac.milton.montufar@gmail.com

${ }^{2}$ Instituto Nacional de Pesca, Ecuador, uavendano@institutopesca.gob.ec

${ }^{3}$ Instituto Nacional de Pesca, Ecuador, wruiz@institutopesca.gob.ec

${ }^{4}$ Instituto Nacional de Pesca, Ecuador, xicaza@institutopesca.gob.ec

${ }^{5}$ Training Altatierra\&Asociados, Ecuador, cem.ellibertador@gmail.com
} 


\section{Resumen.}

La maricultura es un sector en desarrollo que está asociado a diversos riesgos de trabajar en el mar. En Ecuador no se ha realizado estudios exhaustivos de estos peligros, y existe una gran incertidumbre sobre su alcance. La pregunta que se abordó en esta investigación fue: ¿Qué se sabe sobre los riesgos ocupacionales para los maricultores artesanales? Mediante entrevistas abiertas y visitas realizadas a todas las Cooperativas de Pescadores Artesanales que se dedican a esta actividad se identificó y valoró los riesgos laborales utilizando el índice de riesgo de seguridad ocupacional. En este análisis, las causas de muerte incluyen ahogamiento, y lesión cerebral fatal.; las causas de incapacidad se asocian con lesiones dorsolumbares; las causas de lesiones graves incluyen caídas en la embarcación, riesgos asociados al buceo, proyecciones de partículas a los ojos, cortes o abrasión en la piel, y enfermedades en la piel provocadas por la exposición prolongada a la radiación solar sin protección adecuada; y en las causas de lesiones moderadas se determinaron afecciones dermatológicas provocados por contacto directo con organismos acuáticos.

Palabras Claves: Maricultura, Prevención, Riesgo, Índice, Ocupacional

\section{Introducción .}

La maricultura es una actividad relativamente nueva en Ecuador, regulada desde el 2013 mediante Acuerdo Ministerial 458 con el Instructivo para el Ordenamiento y Control de Concesiones para Maricultura en el Ecuador. Actualmente el Acuerdo Ministerial 458 fue derogado por el Acuerdo Ministerial 023. La maricultura se presenta como una nueva actividad productiva para aliviar la presión de extracción de la pesca, dando respuesta al incremento de la demanda de productos marinos local e internacional.

Ecuador tiene aproximadamente 640 kilómetros de línea de costa, con muchos puntos protegidos que podrían ser utilizados para el desarrollo de los cultivos marinos y condiciones oceanográficas que dan un gran potencial para esta actividad. Actualmente en Ecuador se cultiva en maricultura macroalgas (Kappaphycus alvarezzi) y ostras (Crassostrea gigas) a nivel artesanal, y cobia (Rachycentron canadum) a nivel industrial con una producción promedio de 5.2, 7.6 y 316.5 toneladas respectivamente.

Existen varios proyectos de cultivo de ostras ejecutándose en la provincia de Santa Elena; en la comuna La Entrada Asociación ASMACUIAMAR, comuna El Palmar COPROACMAR, comuna El Real Cooperativa Puerto Real Alto y la Cooperativa Nuevos Horizontes en la parroquia Chanduy. El cultivo de macroalgas lo realiza la Cooperativa de Producción Pesquera Santa Rosa de Salinas, en la caleta pesquera Santa Rosa del cantón Salinas, provincia de Santa Elena. 
En Ecuador no existe información previa publicada respecto a prevención de riesgos laborales en maricultura. El objetivo de esta investigación es identificar riesgos laborales en la maricultura artesanal de Ecuador y proponer medidas preventivas que minimicen los mismos. Para identificar los riesgos laborales se realizó un seguimiento a los procesos de cultivo en todos los lugares de producción.

Es importante analizar este problema porque los maricultores artesanales tienen pocos recursos económicos para contratar personal capacitado que elabore el Reglamento de Seguridad y Salud de los trabajadores, además que en el país existen pocos profesionales expertos en maricultura y seguridad ocupacional. La hipótesis es que la maricultura es una actividad riesgosa en materia de seguridad ocupacional, por lo tanto es necesario identificar y prevenir los posibles riesgos laborales de la actividad.

Esta investigación pretende guiar sistemáticamente la evaluación de riesgos en una instalación acuícola en el mar, y luego ofrecer charlas a los maricultores artesanales para que puedan prevenir accidentes laborales.

La ergonomía es la disciplina científica que se ocupa de la comprensión fundamental de las interacciones entre los seres humanos y los otros componentes de un sistema. Es la disciplina que aplica principios teóricos, datos y métodos para optimizar el bienestar de las personas y el rendimiento global del sistema.

La ergonomía contribuye a la planificación, evaluación, concepción y evaluación de las tareas, trabajos, productos, organizaciones, entorno y sistemas para hacerlos compatibles con las necesidades, capacidades y limitaciones de las personas (Wilson, 2013). Para poder aplicar el concepto de mejora continua dentro del Sistema de Gestión de Seguridad se recomienda realizar una evaluación ergonómica de los puestos de trabajo en cada proceso mencionado en esta investigación.

\section{Importancia del Problema.}

En Ecuador esta investigación es la primera sobre prevención de Riesgos Laborales en Maricultura Artesanal aplicada al sector, es necesario resolver este problema social para que los maricultores artesanales puedan mantener una adecuado Sistema de Seguridad y Salud Ocupacional. A nivel mundial son comunes los accidentes laborales, lesiones e incluso muertes en el ámbito laboral de esta actividad, los cuales generablemente no son documentados ni publicados, dificultando el levantamiento de datos y la investigación en esta área.

En Ecuador las empresas acuícolas tienen un valor promedio de sustentabilidad social próximo a la sostenibilidad social, sin embargo es necesario mejorar aspectos de la sostenibilidad social como son las medidas de prevención de riesgos laborales (López, 2016) 


\section{Metodología.}

Como referencia se tomó la normativa nacional Reglamento de seguridad y salud de los trabajadores del Ministerio de Trabajo Modificado el 21-feb.-2003, Decreto Ejecutivo 2393, y la norma internacional OHSAS 18001:2007, para levantar la información se realizaron dos visitas a cada cooperativa de cultivo de ostras y macroalgas durante el año 2017, con el fin de identificar y valorar los riesgos laborales en todos los procesos de cultivo mediante entrevistas abiertas y observaciones en los lugares de producción.

Figura 1. Proceso de gestión de riesgo.

\begin{tabular}{|l|}
\hline Identificación de riesgos \\
\hline$\square$ \\
\hline $\begin{array}{l}\text { Análisis de probabilidad de } \\
\text { riesgos }\end{array}$ \\
$\square$ \\
\hline Análisis de gravedad de riesgos \\
\hline \\
\hline Índice de riesgo \\
\hline \\
\hline Medida de prevención \\
\hline
\end{tabular}

Fuente: Autores, Ecuador, 2018.

Para procesar la información se siguió el proceso de gestión de riesgo descrito en la Figura 1. Una vez recogidos los datos concernientes a la identificación de los riesgos existentes, se realizó la evaluación de los mismos en función de los dos factores fundamentales de incidencia como son probabilidad de producir un accidente, y consecuencias de la lesión potencial que produciría.

Para determinar la probabilidad de ocurrencia de un riesgo, se elaboró una matriz descrita en la tabla 1, la cual cuenta con una definición cualitativa y otra cuantitativa para asignar un valor en la escala del 1 al 5, siendo 5 la probabilidad de ocurrencia más alta y 1 la más baja.

Tabla 1. Matriz de probabilidad de los riesgos de seguridad operacional.

\begin{tabular}{llll}
\hline Probabilidad & Definición Cualitativa & Cuantitativa & Valor \\
\hline Muy alta & Probable que ocurra muchas veces & Una vez al año & 5 \\
Alta & Probable que ocurra algunas veces & Cada 1 o 5 años & 4 \\
Ocasional & Improbable pero prosible que ocurra & Cada 5 o diez años & 3 \\
Baja & Muy improbable que ocurra & Mayor a diez años & 2 \\
Muy baja & Casi inconcebible que el suceso ocurra & Nunca & 1 \\
\hline
\end{tabular}

Fuente: Autores, Ecuador, 2018.

Para determinar la gravedad del riesgo, se elaboró la matriz descrita en la tabla 2 , en esta matriz la gravedad es directamente proporcional a la consecuencia, siendo la muerte la 
consecuencia más alta, y lesiones leves la consecuencia más baja. Para asignarle un valor se utilizaron las primeras cinco letras del alfabeto, siendo A el grado más alto de severidad y $\mathrm{E}$ el grado más bajo.

Tabla 2. Matriz de gravedad (severidad) de los riesgos de seguridad operacional.

\begin{tabular}{lll}
\hline Severidad & Consecuencia & Valor \\
\hline Catastrófico & Muerte & $\mathrm{A}$ \\
Mayor & Lesiones que provocan incapacidad & $\mathrm{B}$ \\
Moderado & Lesiones graves & $\mathrm{C}$ \\
Leve & Lesiones moderadas & $\mathrm{D}$ \\
Trivial & Lesiones leves & $\mathrm{E}$
\end{tabular}

Fuente: Autores, Ecuador, 2018.

Para poder clasificar los riesgos laborales en función de la severidad y probabilidad de que ocurran, se desarrolló el índice de seguridad operacional de la tabla 3. La combinación de la severidad y la probabilidad dieron como resultado cuatro niveles de índices de riesgo: intolerable, alto, moderado, tolerable y bajo. Los valores de los índices de riesgo del nivel intolerable fueron 5A, 5B, 4A, y 4B; los valores de los índices de riesgo del nivel alto fueron $5 \mathrm{C}, 4 \mathrm{C}, 3 \mathrm{~A}, 3 \mathrm{~B}$ y $2 \mathrm{~A}$; valores de los índices de riesgo del nivel moderado fueron $5 \mathrm{D}, 5 \mathrm{E}, 4 \mathrm{D}, 4 \mathrm{E}, 3 \mathrm{C}, 2 \mathrm{~B}, 2 \mathrm{C}$ y $1 \mathrm{~A}$; y valores de los índices de riesgo del nivel bajo fueron 2E, 1D, y 1E. Siendo el índice de riesgo de seguridad operacional 5A de nivel intolerable el de mayor riesgo y $1 \mathrm{E}$ de nivel bajo el de menor riesgo.

Tabla 3. Matriz de evaluación (índice) de riesgo de seguridad operacional.

\begin{tabular}{|c|c|c|c|c|c|}
\hline \multirow[b]{2}{*}{ PROBABILIDAD } & \multicolumn{5}{|c|}{ SEVERIDAD } \\
\hline & $\begin{array}{l}\text { Catastrófico } \\
\text { (A) }\end{array}$ & $\begin{array}{l}\text { Mayor } \\
\text { (B) }\end{array}$ & $\begin{array}{l}\text { Moderado } \\
\text { (C) }\end{array}$ & $\begin{array}{l}\text { Leve } \\
\text { (D) }\end{array}$ & $\begin{array}{l}\text { Insignificante } \\
\text { (E) }\end{array}$ \\
\hline $\begin{array}{l}\text { Muy alta } \\
\text { (5) }\end{array}$ & $\begin{array}{l}\text { Intolerable } \\
\left(I_{R}=5 A\right)\end{array}$ & $\begin{array}{l}\text { Intolerable } \\
\left(I_{R}=5 B\right)\end{array}$ & $\begin{array}{l}\text { Alto } \\
\left(I_{R}=5 C\right)\end{array}$ & $\begin{array}{l}\text { Moderado } \\
\left(\mathrm{I}_{\mathrm{R}}=5 \mathrm{D}\right)\end{array}$ & $\begin{array}{l}\text { Moderado } \\
\left(I_{R}=5 E\right)\end{array}$ \\
\hline $\begin{array}{l}\text { Alta } \\
\text { (4) }\end{array}$ & $\begin{array}{l}\text { Intolerable } \\
\left(I_{R}=4 A\right)\end{array}$ & $\begin{array}{l}\text { Intolerable } \\
\left(I_{R}=4 B\right)\end{array}$ & $\begin{array}{l}\text { Alto } \\
\left(I_{R}=4 C\right)\end{array}$ & $\begin{array}{l}\text { Moderado } \\
\left(I_{R}=4 D\right)\end{array}$ & $\begin{array}{l}\text { Moderado } \\
\left(I_{R}=4 E\right)\end{array}$ \\
\hline $\begin{array}{l}\text { Ocasional } \\
\text { (3) }\end{array}$ & $\begin{array}{l}\text { Alto } \\
\left(I_{R}=3 A\right)\end{array}$ & Alto $\left(I_{R}=3 B\right)$ & $\begin{array}{l}\text { Moderado } \\
\left(I_{R}=3 C\right)\end{array}$ & $\begin{array}{l}\text { Tolerable } \\
\left(I_{R}=3 D\right)\end{array}$ & $\begin{array}{l}\text { Tolerable } \\
\left(I_{R}=3 E\right)\end{array}$ \\
\hline $\begin{array}{l}\text { Baja } \\
(2)\end{array}$ & $\begin{array}{l}\text { Alto } \\
\left(I_{R}=2 A\right)\end{array}$ & $\begin{array}{l}\text { Moderado } \\
\left(I_{R}=2 B\right)\end{array}$ & $\begin{array}{l}\text { Moderado } \\
\left(I_{R}=2 C\right)\end{array}$ & $\begin{array}{l}\text { Tolerable } \\
\left(I_{R}=2 D\right)\end{array}$ & Bajo $\quad\left(I_{R}=2 E\right)$ \\
\hline $\begin{array}{l}\text { Muy baja } \\
\text { (1) }\end{array}$ & $\begin{array}{l}\text { Moderado } \\
\left(I_{R}=1 \mathrm{~A}\right)\end{array}$ & $\begin{array}{l}\text { Tolerable } \\
\left(\mathrm{I}_{\mathrm{R}}=1 \mathrm{~B}\right)\end{array}$ & $\begin{array}{l}\text { Tolerable } \\
\left(I_{R}=1 C\right)\end{array}$ & $\begin{array}{l}\text { Bajo } \\
\left(I_{R}=1 D\right)\end{array}$ & Bajo $\quad\left(I_{R}=1 E\right)$ \\
\hline
\end{tabular}

Fuente: Autores, Ecuador, 2018 
Una vez clasificados los riesgos laborales según el índice de seguridad operacional, mediante la tabla 4 se propuso los criterios sugeridos de acción de prevención de acuerdo a la tolerabilidad del riesgo.

Tabla 4. Matriz de tolerabilidad del riesgo de seguridad operacional.

\begin{tabular}{|c|c|c|}
\hline $\begin{array}{l}\text { ÍNDICE } \\
\text { DE } \\
\text { RIESGO }\end{array}$ & TOLERABILIDAD & CRITERIOS SUGERIDOS \\
\hline $\begin{array}{l}5 \mathrm{~A}, 5 \mathrm{~B}, 4 \mathrm{~A}, \\
4 \mathrm{~B}\end{array}$ & Riesgo intolerable & $\begin{array}{l}\text { No hay que permitir ninguna operación hasta que se hayan } \\
\text { implementado medidas de control adecuadas para reducir el riesgo a un } \\
\text { nivel aceptable. Se requiere la aprobación del máximo nivel de la } \\
\text { administración. }\end{array}$ \\
\hline $\begin{array}{l}5 \mathrm{C}, 4 \mathrm{C}, 3 \mathrm{~A} \\
3 \mathrm{~B}, 2 \mathrm{~A}\end{array}$ & Alto riesgo & $\begin{array}{l}\text { Precaución. Hay que asegurarse de que la evaluación de riesgos se ha } \\
\text { completado satisfactoriamente y que los controles preventivos } \\
\text { declarados están implementados. Aprobación de la evaluación de } \\
\text { riesgos por parte de la administración superior antes del inicio de la } \\
\text { operación o proceso }\end{array}$ \\
\hline $\begin{array}{l}5 \mathrm{D}, 5 \mathrm{E}, 4 \mathrm{D} \\
4 \mathrm{E}, 3 \mathrm{C}, 2 \mathrm{~B} \\
2 \mathrm{C}, 1 \mathrm{~A}\end{array}$ & Riesgo moderado & Aplicar medidas de prevención de riesgos. \\
\hline $\begin{array}{l}3 \mathrm{D}, 3 \mathrm{E}, 2 \mathrm{D}, \\
1 \mathrm{~B}, 1 \mathrm{C}\end{array}$ & Bajo tolerable & Las medidas prevención de riesgos es opcional. \\
\hline $2 \mathrm{E}, 1 \mathrm{D}, 1 \mathrm{E}$ & Riesgo bajo & No es necesario medidas de prevención de riesgos. \\
\hline
\end{tabular}

Fuente: Autores, Ecuador, 2018.

\section{Resultados.}

En las figura 2 se desarrolló el diagrama de flujo del proceso de cultivo de macroalgas en Ecuador para poder analizar el índice de riesgo de acuerdo al proceso de cada actividad. El cultivo inicia con la instalación del sistema de fondeo y las balsas para la siembra de talos, la instalación del sistema de fondeo sólo se realiza una sola vez. El tiempo promedio del ciclo de cultivo desde la siembra de talos hasta la cosecha es de 35 días. En los muestreos biométricos se realizan mediciones de talla y peso.

Para el mantenimiento del sistema del fondeo se realizan maniobras con la embarcación para tensionar el long line principal donde va a ir instaladas las balsas. Además se realizan labores de mantenimiento submarinas mediante inmersiones de buceo para revisar los pesos muertos, anillas y cabos. Como la macroalga Kappaphycus alvarezii es una especie no nativa, también se realizan muestreos inmersiones para ambientales mediante cuadrantes dentro del área de cultivo.

Luego de la cosecha la macroalga se limpia separando el fouling adherido mediante lavado, una parte se destina como semilla para volver a sembrar y otra porción va a los secadores 
solares en la playa. Una vez seca la macroalga es almacenada en bodegas adecuadas con pallets y con circulación de aire para controlar la humedad del ambiente. El proceso de transporte y venta todavía no se lleva a cabo en el país porque todavía falta completar el volumen requerido para exportar la materia prima seca destinada para extracción de carragenina.

Figura 2. Diagrama de flujo del proceso de cultivo de macroalgas (Kappaphycus alvarezii).

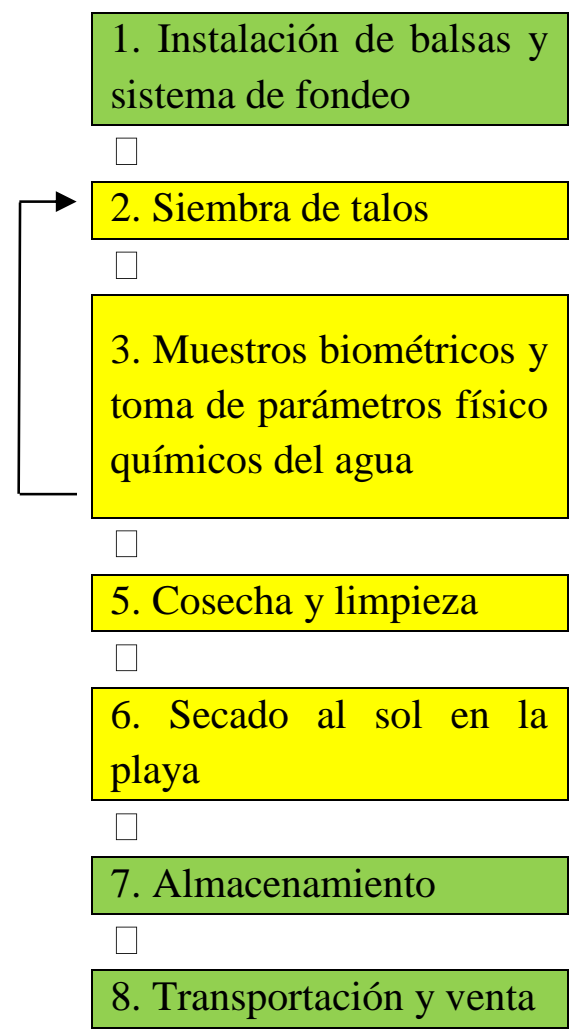

Actividad rutinaria

Actividad no rutinaria

Fuente: Autores, Ecuador, 2018.

En las figura 3 se desarrolló el diagrama de flujo del proceso de cultivo de Ostra del Pacífico (Crassostrea gigas) para poder analizar el índice de riesgo de acuerdo al proceso de cada actividad. El cultivo inicia con la instalación del sistema de fondeo y long lines para la siembra de las linternas, la instalación del sistema de fondeo sólo se realiza una sola vez. El tiempo promedio del ciclo de cultivo desde la siembra de las linternas hasta la cosecha es de 8 meses. Durante el cultivo se realizan dos desdobles para reducir el $50 \%$ la densidad de cultivo en cada piso de las linternas, en este proceso se cambian las linternas por un ojo de malla mayor. En los muestreos biométricos se realizan mediciones de talla y 
peso, en este proceso también se realiza el mantenimiento de linternas retirando el fouling adherido a las redes y a los long lines.

Para el mantenimiento del sistema del fondeo se realizan maniobras con la embarcación para tensionar el long line principal donde van a ir instaladas las linternas. Además se realizan labores de mantenimiento submarinas mediante inmersiones de buceo para revisar los pesos muertos, anillas y cabos.

Generalmente las cosechas se realizan bajo pedido, luego de la cosecha las ostras se limpian eliminando los epibiontes manualmente o con agua a presión. Las ostras se venden vivas in situ o pueden ser transportadas a Guayaquil, Quito, o Cuenca.

Figura 3. Diagrama de flujo del proceso de cultivo de ostras (Crassostrea gigas).

\begin{tabular}{|l}
\hline 9. Instalación de líneas \\
suspendidas y sistema de \\
fondeo
\end{tabular}

\section{Siembra de semilla}

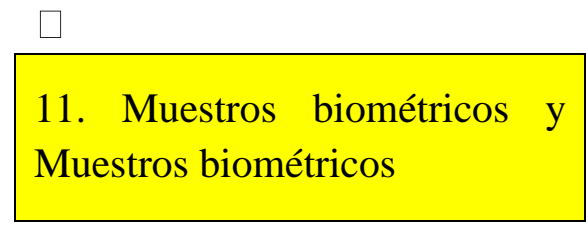

12. Mantenimiento de líneas suspendidas, linternas, y sistema de fondeo

\section{Cosecha y limpieza}

\section{Transportación y venta}

Actividad rutinaria

Actividad no rutinaria

Fuente: Autores, Ecuador, 2018.

El Índice de Riesgo Laboral de nivel intolerable se detalla en el Anexo 1, las principales causas identificadas en este nivel fueron riesgo de lesiones dorsolumbares por exceso de carga en la instalación de pesos muertos, por subir linternas a la embarcación, por trasladar sacos de macroalgas cosechadas desde la embarcación a la playa, por trasladar tanques de combustible desde el muelle a la embarcación, por posturas de trabajo inadecuadas, por repetición en el manejo de cargas, y por realizar muestreos de biomasa en líneas o mangas de macroalgas en la embarcación. 
El Índice de Riesgo Laboral de nivel intolerable se detalla en el Anexo 1, las principales causas identificadas en este nivel fueron riesgo de lesiones dorsolumbares por exceso de carga en la instalación de pesos muertos, por subir linternas a la embarcación, por trasladar sacos de macroalgas cosechadas desde la embarcación a la playa, por trasladar tanques de combustible desde el muelle a la embarcación, por posturas de trabajo inadecuadas, por repetición en el manejo de cargas, y por realizar muestreos de biomasa en líneas o mangas de macroalgas en la embarcación.

El Índice de Riesgo Laboral de nivel alto se desarrolló en el Anexo 2, las principales causas identificadas en este nivel fueron riesgo en el buceo provocado por quedar atrapado en redes de pesca abandonadas, y riesgo ahogamiento por vuelco de la embarcación

El Índice de Riesgo Laboral de nivel moderable se desarrolló en el Anexo 3, las principales causas identificadas en este nivel fueron riesgo de caída en la embarcación por falta de orden en la cubierta, por llevar calzado inadecuado o no tener calzado, por choques o reducciones de velocidad bruscas ocasionadas por objetos no señalizados o a la deriva, riesgo de caída al subir a la embarcación, riesgo en el buceo provocado por cortes con objetos punzantes como rocas, riesgo en el buceo provocado por hipoacusia, riesgos de afecciones dermatológicas provocados por exposición directa prolongada con el sol.

Además de riesgo de proyección de partículas a los ojos y abrasión de la piel debido al uso de agua a presión mientras lava las mallas provocado por la falta de concentración, planificación o uso de equipo de protección personal, riesgo de cortes provocado por elementos con filo, riesgo de accidentes ocasionados por piratas en el mar, riesgo de caída al mar por mar agitado, riesgo de caída en la embarcación por falta de concentración del trabajador al caminar por la cubierta u otras superficies, riesgo de incendio en la embarcación debido al manejo inadecuado del combustible utilizado, riesgo de quedar a la deriva por recalentamiento del motor fuera de borda debido a la mantención u operación inadecuada, Riesgo en el buceo provocado por narcosis, por sobreesfuerzos al equiparse en la embarcación y por lesión cerebral fatal al golpearse con una roca, riesgo de quedar atrapado entre la embarcación y otro bote, balsa, o muelle.

El Índice de Riesgo Laboral de nivel tolerable se detalla en el Anexo 4, las principales causas identificadas en este nivel fueron riesgo de afecciones dermatológicas provocados por contacto directo con organismos acuáticos, riesgo de cortes provocado al realizar maniobras para tensionar las líneas de los cultivos suspendidos en la cuerda al contacto con las manos sin guantes pueden causar abrasión o cortes en las manos, riesgo en el buceo provocado por cortes al subir o bajar por el la línea de vida, o cabo que lleva al peso muerto, ésta puede tener broma y realizar cortes en las manos sin guantes, riesgo en el buceo provocado por seres vivos como lobos marinos, riesgo en el buceo provocado por diferencias de presión, y riesgo de caída en la embarcación por falta de iluminación en faenas nocturnas. 
Finalmente en el Índice de Riesgo Laboral de nivel bajo se detalla en el Anexo 5, las principales causas identificadas en este nivel fueron riesgos de afecciones dermatológicas provocados por contacto con lubricantes y desinfectantes.

Para determinar la predominancia de los riesgos laborales en la maricultura artesanal de Ecuador en función de cantidad, se calculó el porcentaje de causas de riesgos laborales por nivel de Índice de Riesgo Laboral presentados en la figura 4, el resultado fue $51 \%$ de predominancia para el Índice de Riesgo Laboral de nivel moderable, 20\% para el nivel intolerable, $17 \%$ para el nivel moderable, y el $6 \%$ para los niveles alto y bajo.

Figura 4.Niveles de Índice de Riesgo Laboral en la Maricultura Artesanal de Ecuador.

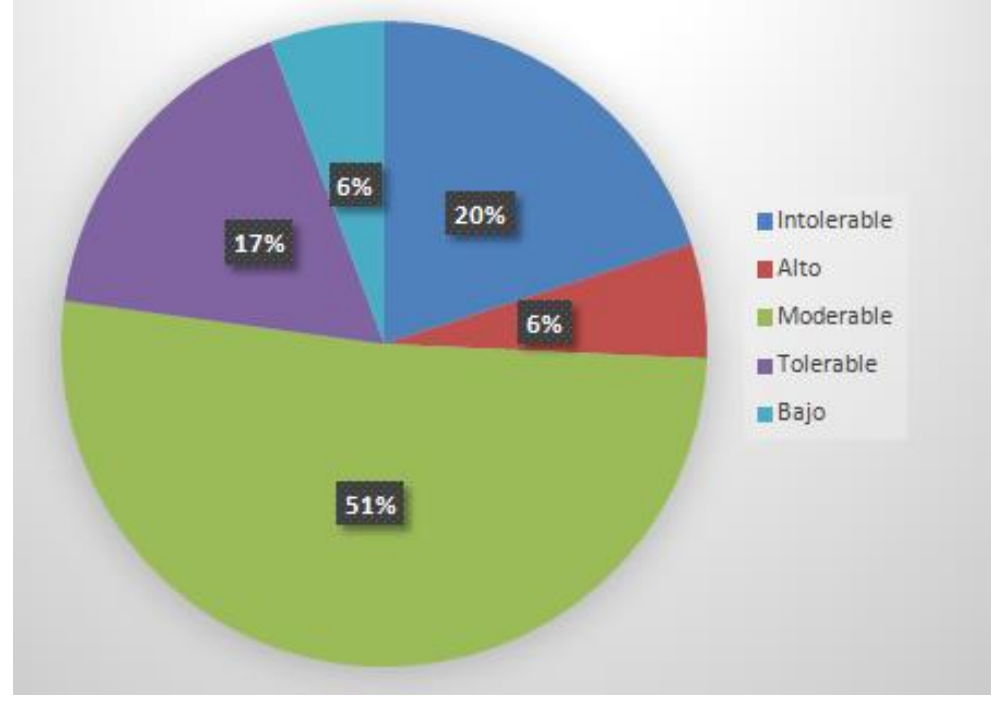

Fuente: Autores, Ecuador, 2018.

\section{Discusión.}

La identificación y evaluación de los riesgos en las actividades relacionadas a la maricultura artesanal en Ecuador confirma la hipótesis que la maricultura es una actividad riesgosa en materia de seguridad ocupacional.

Los riesgos en maricultura artesanal tales como riesgos de lesiones dorsolumbares y riesgos en el buceo son comunes a nivel mundial. Existen varias variables que influyen al momento de identificar los riesgos relacionados para esta actividad en diferentes países:

La primera variable es el nivel económico de la empresa, mientras mayor sea la cantidad de recursos financieros es más probable que la empresa pueda adquirir tecnología, equipos y materiales necesarios para evitar accidentes laborales, además de contratar profesionales para diseñar y poner en práctica el Sistema de Seguridad y Salud Ocupacional. Países de 
primer mundo generalmente tienen más presupuesto y recursos tecnológicos que países de tercer mundo como Ecuador, esto se corrobora en la práctica en el nivel de financiamiento y tecnología de las Cooperativas de Pescadores Artesanales en Ecuador.

La segunda variable es el compromiso de responsabilidad social de la administración de la empresa con los empleados y con la normativa vigente en el país, ésta variable generalmente es directamente proporcional al grado de conocimiento de estos temas de la administración. En Ecuador existe poco conocimiento de los conceptos integrales de responsabilidad social y normativa nacional vigente respecto a Seguridad y Salud Ocupacional.

La tercera variable es el tiempo que tiene la industria establecida en el país, porque de esto depende la infraestructura complementaria necesaria para el desarrollo de la actividad como vendedores de insumos, equipos y materiales adaptados para esta actividad. Mientras mayor tiempo tenga la industria es más probable que sea más grande, y al ser estable en el tiempo la administración pueda realizar las inversiones necesarias para generar sostenibilidad en este aspecto. Además mientras mayor tiempo tiene la industria es más probable que los Sistema de Seguridad y Salud Ocupacional aprobados para la empresa se vayan mejorando cada año y además se contaría con una línea base de accidentes más comunes y sus planes de prevención y contingencia.

En Ecuador se impulsa el cultivo artesanal de Ostras del Pacífico en líneas suspendidas en el mar desde el año 2007 por medio del Viceministerio de Acuacultura y Pesca, como parte del proyecto "Maricultura y piscicultura para el fomento acuícola en el Ecuador" (Montúfar, et al 2013), en Italia los cultivos de moluscos en sistemas suspendidos iniciaron en 1855, en Francia en 1880, en Estados Unidos en 1920, en Japón en 1930, y en España en 1863 (González, 2018), comparado a nivel mundial la maricultura en Ecuador es relativamente nueva, y es cuestión de tiempo para que se iguale con los estándares de otros países que tienen más experiencia en esta industria.

Actualmente las Cooperativas de pescadores artesanales dedicados a esta actividad no cuentan con un Sistema de Seguridad y Salud Ocupacional realizado por un profesional y aprobado por la autoridad correspondiente. Esto se debe a que la actividad todavía se está desarrollando y que su objetivo es generar una fuente de ingreso complementaria, ya que la principal fuente de ingreso directo de estas cooperativas es la pesca.

Esta línea base, contribuye para la elaboración y ejecución del Sistema de Seguridad Industrial de cada cooperativa y de esta manera prevenir accidentes laborales en las organizaciones.

Es preciso realizar un seguimiento de los riesgos que enfrentan los trabajadores en el desarrollo de la maricultura artesanal. Las actividades de seguimiento deben ser diseñadas y 
realizadas por profesionales acreditados como parte del Sistema de Gestión de Seguridad y Salud Ocupacional. Las instalaciones, además, deben llevar un registro de los accidentes y enfermedades laborales, así como de los sucesos y accidentes peligrosos.

Deben adoptarse medidas para reducir a cero el número de accidentes entre los trabajadores del proyecto (ya sean empleados directos o visitas ocasionales), especialmente los accidentes que pueden causar la pérdida de horas de trabajo, diversos niveles de discapacidad e incluso la muerte.

Las áreas físicas de trabajo deberían ser inspeccionadas como mínimo cada tres meses por los mandos intermedios, reflejando documentalmente las deficiencias detectadas y las acciones pertinentes adoptadas.

Los directores de las unidades funcionales, como mínimo semestralmente, deberían visitar los lugares de trabajo para realizar inspecciones de seguridad, acompañados de los mandos intermedios.

\section{Anexos.}

Anexo 1. Índice de riesgo laboral de nivel intolerable en la Maricultura Artesanal de Ecuador

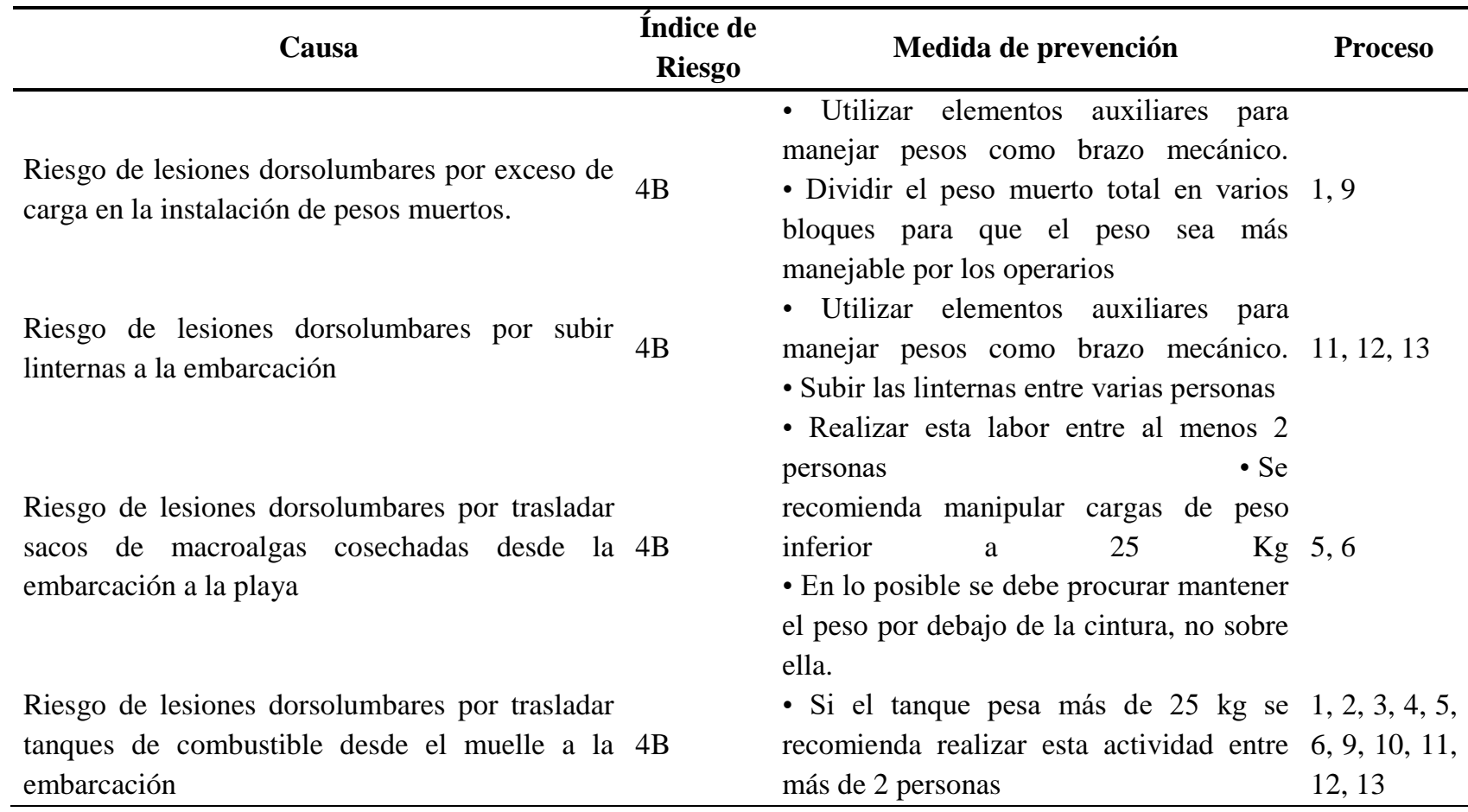

\section{Elaborado por: Grupo de Investigación}




\begin{tabular}{|c|c|c|c|}
\hline Causa & $\begin{array}{c}\text { Índice de } \\
\text { Riesgo }\end{array}$ & Medida de prevención & Proceso \\
\hline $\begin{array}{l}\text { Riesgo de lesiones } \\
\text { dorsolumbares por posturas de } \\
\text { trabajo inadecuadas }\end{array}$ & $4 \mathrm{~B}$ & $\begin{array}{l}\text { - Capacitar al trabajador } \\
\text {-Posibilitar cambios de postura } \\
\text {-Se debe mantener el peso con los brazos lo más } \\
\text { apegado al cuerpo, no realizar extensiones de brazo con } \\
\text { carga pesada. } \\
\text { - No girar sólo la cintura para trasladar cargas, se girar } \\
\text { todo el cuerpo, empezando por los pies. } \\
\text { - Al levantar una carga se debe flexionar las rodillas (no } \\
\text { flexionar el tronco como es habitual). } \\
\text { - A la hora de levantar la carga se debe colocar frente a } \\
\text { ella, colocando los pies abiertos y ligeramente } \\
\text { desalineados para asegurar la estabilidad lateral y } \\
\text { frontal. }\end{array}$ & $\begin{array}{l}1,2,3,4,5 \\
6,7,9,10 \\
11,12,13,14\end{array}$ \\
\hline $\begin{array}{l}\text { Riesgo de lesiones } \\
\text { dorsolumbares por repetición } \\
\text { en el manejo de cargas. }\end{array}$ & 4B & $\begin{array}{l}\text { - Generar procedimiento de trabajo en el que se incluya } \\
\text { el tiempo suficiente de reposo fisiológico recomendado } \\
\text { para este tipo de actividad }\end{array}$ & $\begin{array}{l}1,2,3,4,5 \\
6,7,9,10 \\
11,12,13,14\end{array}$ \\
\hline $\begin{array}{l}\text { Riesgo de lesiones } \\
\text { dorsolumbares al realizar } \\
\text { muestreos de biomasa en líneas } \\
\text { o mangas de macroalgas en la } \\
\text { embarcación }\end{array}$ & 4B & - Los operarios deben tener una aptitud física adecuada. & 4 \\
\hline
\end{tabular}

Fuente: Autores, Ecuador, 2018.

Anexo 2. Índice de riesgo laboral de nivel alto en la Maricultura Artesanal de Ecuador.

\begin{tabular}{|c|c|c|c|}
\hline Causa & $\begin{array}{c}\text { Índice de } \\
\text { Riesgo }\end{array}$ & Medida de prevención & Proceso \\
\hline $\begin{array}{l}\text { Riesgo en el buceo provocado } \\
\text { por quedar atrapado en redes de } \\
\text { pesca abandonadas }\end{array}$ & $2 \mathrm{~A}$ & $\begin{array}{l}\text { - Tener licencia de buzo profesional (open water) } \\
\text { - Nunca bucear solo/a y menos aún con alcohol residual en } \\
\text { el cuerpo o en caso de sentir alguna indisposición } \\
\text { - Llevar cuchillo de buceo en caso de quedar atrapado en } \\
\text { alguna red abandonada. }\end{array}$ & $1,4,9,12$ \\
\hline $\begin{array}{l}\text { Riesgo ahogamiento por vuelco } \\
\text { de la embarcación }\end{array}$ & $2 \mathrm{~A}$ & $\begin{array}{l}\text { - Evitar salir con el mar agitado porque la embarcación se } \\
\text { puede virar en la zona de rompiente. } \\
\text { - Revisar las condiciones oceánicas y planificar de } \\
\text { acuerdos a los periodos de aguajes y quiebra } \\
\text { - Utilización de muelles adecuados para evitar atravesar la } \\
\text { zona de rompientes de olas. } \\
\text { - Llevar chalecos salvavidas para cada uno de los } \\
\text { tripulantes de acuerdo a la normativa SOLAS }\end{array}$ & $\begin{array}{c}1,2,3,4,5 \\
9,10,11 \\
12,13\end{array}$ \\
\hline
\end{tabular}

Fuente: Autores, Ecuador, 2018 
Anexo 3. Índice de riesgo laboral de nivel moderable en la Maricultura Artesanal de Ecuador.

\section{Causa}

Riesgo de caída en la embarcación por calzado inadecuado o no llevar calzado

Riesgo de caída en la embarcación por choques o reducciones de velocidad bruscas ocasionadas por objetos no señalizados o a la deriva como redes de pesca que pueden quedar enredadas en la hélice del motor fuera de borda

Riesgo de caída al subir a la embarcación

$3 \mathrm{C}$

Riesgo en el buceo provocado por cortes con objetos punzantes como rocas

de

\section{Índice}

Riesgo

- Con el objeto de prevenir

tropezones, todos los aparejos y

$1,2,3,4$, cabos de la embarcación deben

$5,6,9$, quedar envueltas en la cubierta, o 10,11 , guardadas en bodegas 12,13 implementadas en la embarcación.

$1,2,3,4$

- Usar botas de caucho o calzado $5,6,9$, con suelas antideslizantes 10,11 , 12,13

- No ir a exceso de velocidad

- La persona encargada de manejar el motor fuera de borda $\quad 1,2,3,4$, debe procurar tener la vista en el $\quad 5,6,9$, horizonte, manteniendo la 10,11 , concentración en la ruta, sin 12,13 distraerse en conversaciones con los tripulantes u otras actividades

- Precaución en el acceso a la embarcación y las operaciones que se lleven a bordo, para evitar caídas al mar

$1,2,3,4$ $5,9,10$, $11,12,13$

3C - Utilizar el traje de buzo completo 1, 4, 9, 12

\begin{tabular}{|c|c|c|c|}
\hline Causa & $\begin{array}{c}\text { Índice de } \\
\text { Riesgo }\end{array}$ & Medida de prevención & Proceso \\
\hline Riesgo en el buceo provocado por hipoacusia & $3 \mathrm{C}$ & $\begin{array}{l}\text { - Si se tiene molestias en los oídos al } \\
\text { ascender y descender se sugiere tragar } \\
\text { saliva tapar la salida del aire por la boca y } \\
\text { nariz mientras espira con fuerza. } \\
\text { - Profilaxis médica contra la otitis externa }\end{array}$ & $1,4,9,12$ \\
\hline
\end{tabular}


Riesgos de afecciones dermatológicas provocados por exposición directa prolongada con el sol

Riesgo de proyección de partículas a los ojos y abrasión de la piel debido al uso de agua a presión mientras lava las mallas provocado por la falta de concentración, planificación o uso de equipo de protección personal

Riesgo de cortes provocado por elementos con filo (cuchillo, ancla, elementos orgánicos o inorgánicos en la superficie de la embarcación) debido a falta de concentración del operario o al movimiento de la embarcación

Riesgo de accidentes ocasionados por piratas en el mar
- Utilizar bloqueador solar, ropa adecuada

3C para el trabajo (algodón, con filtro UV) de tonos claros, gorra.

$1,2,3,4,5$, $6,9,10,11$, 12,13

- Capacitar al trabajador en el lavado de mallas.

- Planificar este tipo de actividades

3C - Usar elementos de protección personal

4 adecuados (lentes, guantes, calzado de seguridad)

- Generar procedimiento de trabajo.

$3 \mathrm{C}$

- Utilizar elementos de protección personal.

$1,2,3,4,5$,

$6,9,10,11$,

12,13

- Evitar contacto directo en caso de encuentro con piratas, conocimiento de acciones evasivas con la embarcación. - Llevar botiquín de primeros auxilios
1, 2, 3, 4, 5, 9, 10, 11, 12,13

\begin{tabular}{|c|c|c|c|}
\hline Causa & $\begin{array}{c}\text { Índice de } \\
\text { Riesgo }\end{array}$ & Medida de prevención & Proceso \\
\hline $\begin{array}{l}\text { Riesgo de caída al mar por mar } \\
\text { agitado }\end{array}$ & $2 \mathrm{C}$ & $\begin{array}{l}\text { - Para prevenir una caída al mar se debe evitar salir con } \\
\text { mal tiempo, hacerlo con las condiciones ambientales } \\
\text { adecuadas, y llevar puesto el chaleco salvavidas . En } \\
\text { caso de hacerlo de salir con mal tiempo no acercarse a } \\
\text { los bordes de la embarcación y sujetarse mientras está } \\
\text { en movimiento. Actuar preventivamente }\end{array}$ & $\begin{array}{c}1,2,3,4,5 \\
6,9,10,11, \\
12,13\end{array}$ \\
\hline $\begin{array}{l}\text { Riesgo de caída en la } \\
\text { embarcación por falta de } \\
\text { concentración del trabajador al } \\
\text { caminar por la cubierta u otras } \\
\text { superficies }\end{array}$ & $2 \mathrm{C}$ & $\begin{array}{l}\text { - Alimentación adecuada para evitar mareos } \\
\text { - Para subir y bajar por diferentes niveles debe darse la } \\
\text { cara a los peldaños. } \\
\text { - Seguir los procedimientos de trabajo }\end{array}$ & $\begin{array}{c}1,2,3,4,5 \\
6,9,10,11, \\
12,13\end{array}$ \\
\hline $\begin{array}{l}\text { Riesgo de incendio en la } \\
\text { embarcación debido al manejo } \\
\text { inadecuado del combustible } \\
\text { utilizado. }\end{array}$ & $2 \mathrm{C}$ & $\begin{array}{l}\text { - Mantener bajo control todas las fuentes de calor o de } \\
\text { combustibles. } \\
\text { - Mantener el orden y el aseo en todos los lugares de } \\
\text { trabajo. }\end{array}$ & $\begin{array}{c}1,2,3,4,5 \\
9,10,11 \\
12,13\end{array}$ \\
\hline
\end{tabular}


- Efectuar mantenimiento periódico al motor fuera de borda.

Riesgo de quedar a la deriva por recalentamiento del motor fuera de borda debido a la mantención u operación inadecuada
- Es recomendable que la persona que maneje el motor fuera de borda sea debidamente capacitada en esta 1, 2, 3, 4, 5, 2C actividad y tenga experiencia previa.

$9,10,11$,

- Revisar la cantidad adecuada de combustible antes de

12,13 salir para no quedar a la deriva

- Llevar una radio con frecuencia o un teléfono

satelital. $\quad$ Llevar un par de remos de emergencia

\begin{tabular}{|c|c|c|c|}
\hline Causa & $\begin{array}{l}\text { Índice de } \\
\text { Riesgo }\end{array}$ & Medida de prevención & Proceso \\
\hline $\begin{array}{l}\text { Riesgo en el buceo provocado por narcosis } \\
\text { nitrogenada }\end{array}$ & $2 \mathrm{C}$ & $\begin{array}{l}\text { - En caso de tener la sensación de mareo } \\
\text { o de estar borracho buceando es mejor } \\
\text { ascender respetando la tabla de } \\
\text { descompresión }\end{array}$ & $1,4,9,12$ \\
\hline $\begin{array}{l}\text { Riesgo en el buceo provocado por } \\
\text { sobreesfuerzos al equiparse en la embarcación }\end{array}$ & $2 \mathrm{C}$ & $\begin{array}{l}\text { - Es recomendable que hayan } 2 \text { personas } \\
\text { para equiparse y para subir a la } \\
\text { embarcación una vez finalizado el buceo. }\end{array}$ & $1,4,9,12$ \\
\hline $\begin{array}{l}\text { Riesgo de quedar atrapado entre la embarcación } \\
\text { y otro bote, balsa, o muelle. }\end{array}$ & $2 \mathrm{C}$ & - Realizar el curso OMI & $\begin{array}{c}1,2,3,4,5 \\
9,10,11 \\
12,13\end{array}$ \\
\hline $\begin{array}{l}\text { Riesgo en el buceo provocado por lesión } \\
\text { cerebral fatal al golpearse con una roca }\end{array}$ & $1 \mathrm{~A}$ & - Evitar bucear con corrientes fuertes & $1,4,9,12$ \\
\hline
\end{tabular}

Fuente: Autores, Ecuador, 2018.

Anexo 4. Índice de riesgo laboral de nivel tolerable en la Maricultura Artesanal de Ecuador.

\begin{tabular}{|c|c|c|c|}
\hline Causa & $\begin{array}{c}\text { Índice de } \\
\text { Riesgo }\end{array}$ & Medida de prevención & Proceso \\
\hline $\begin{array}{l}\text { Riesgos de afecciones dermatológicas } \\
\text { provocados por contacto directo con organismos } \\
\text { acuáticos }\end{array}$ & $3 \mathrm{D}$ & $\begin{array}{l}\text { - Usar elementos de protección personal } \\
\text { (guantes, botas, wetsuit completo). }\end{array}$ & $\begin{array}{c}1,2,3,4,5 \\
9,10,11 \\
12,13\end{array}$ \\
\hline $\begin{array}{l}\text { Riesgo de cortes provocado al realizar } \\
\text { maniobras para tensionar las líneas de los }\end{array}$ & & & \\
\hline $\begin{array}{l}\text { cultivos suspendidos en la cuerda al contacto con } \\
\text { las manos sin guantes pueden causar abrasión o } \\
\text { cortes en las manos }\end{array}$ & $3 \mathrm{D}$ & - Utilizar guantes & $1,4,9$ \\
\hline $\begin{array}{l}\text { Riesgo en el buceo provocado por cortes al subir } \\
\text { o bajar por el la línea de vida, o cabo que lleva al } \\
\text { peso muerto, ésta puede tener broma y realizar }\end{array}$ & $3 \mathrm{D}$ & - Utilizar guantes & $1,4,9,12$ \\
\hline
\end{tabular}


Riesgo en el buceo provocado por seres vivos como lobos marinos

Riesgo en el buceo provocado por diferencias de presión.
- Es recomendable evitar acercarse a los

1B lobos marinos porque son animales $1,4,9,12$ salvajes

- Respetar los tiempos durante el ascenso para evitar los síntomas debidos a la descompresión.

$1 \mathrm{~B}$ de bucear (respetar al menos 24 horas).

- Observación estricta de las tablas de descompresión apropiadas antes de realizar la inmersión

\begin{tabular}{llll}
\multicolumn{1}{c}{ Causa } & $\begin{array}{c}\text { Índice de } \\
\text { Riesgo }\end{array}$ & \multicolumn{1}{c}{ Medida de prevención } & \multicolumn{1}{c}{ Proceso } \\
\hline $\begin{array}{l}\text { Riesgo de caída en la embarcación por falta de } \\
\text { iluminación en faenas nocturnas }\end{array}$ & $1 \mathrm{C}$ & $\begin{array}{l}\text { - Señalización correcta que se pueda ver } \\
\text { en la noche }\end{array}$ & $\begin{array}{l}1,2,3,4,5, \\
6,9,10,11, \\
12,13\end{array}$ \\
\hline
\end{tabular}

Fuente: Autores, Ecuador, 2018.

Anexo 5. Índice de riesgo laboral de nivel bajo en la Maricultura Artesanal de Ecuador.

\begin{tabular}{|c|c|c|c|}
\hline Causa & $\begin{array}{c}\text { Índice de } \\
\text { Riesgo }\end{array}$ & Medida de prevención & Proceso \\
\hline $\begin{array}{c}\text { Riesgos de afecciones dermatológicas } \\
\text { provocados por contacto con combustibles o } \\
\text { lubricantes usados en el motor fuera de borda o } \\
\text { brazo mecánico }\end{array}$ & $1 \mathrm{D}$ & $\begin{array}{l}\text { - Generar y seguir procedimientos de } \\
\text { trabajo }\end{array}$ & $\begin{array}{l}1,2,3,4,5 \\
6,9,10,11 \\
\quad 12,13\end{array}$ \\
\hline $\begin{array}{c}\text { Riesgos de afecciones dermatológicas } \\
\text { provocados por uso de cloro concentrado para } \\
\text { limpieza }\end{array}$ & $1 \mathrm{D}$ & $\begin{array}{l}\cdot \text { Generar y seguir procedimientos de } \\
\text { trabajo } \begin{array}{r}\cdot \text { Utilizar otros } \\
\text { elementos de limpieza }\end{array}\end{array}$ & $\begin{array}{l}1,2,3,4,5 \\
6,9,10,11 \\
13\end{array}$ \\
\hline
\end{tabular}

Fuente: Autores, Ecuador, 2018.

\section{Conclusiones.}

- Hasta el momento no se ha reportado accidentes graves en el desarrollo de esta actividad, sin embargo, se ha identificado que las actividades realizadas en la maricultura artesanal generan riesgos laborales en los trabajadores de este sector, 
por lo tanto es necesario que las Cooperativas de Pescadores Artesanales implementen Programas de Ergonomía como parte del Sistema de Gestión de Seguridad y Salud Ocupacional para evitar accidentes laborales en el futuro.

\section{Referencias bibliográficas.}

González, José. (2018). Evolución histórica y situación actual de la acuicultura en el mundo y en España. Recuperado de www.mapama.gob.ec/ministerio/pags/biblioteca/fondo/pdf/29259_8.pdf

- López, Alvarado. (2016). Desarrollo de Indicadores de Sostenibilidad para la Maricultura del Ecuador. Revista Internacional de Investigación y Docencia (RIID), Volumen 1 Número 1, pp 20-32. DOI: http://dx.doi.org/10.19239/riidv1n1p20

- Montúfar, J., \& Montúfar, M. (2013). Análisis del Impacto Socioeconómico del Cultivo en Maricultura de la Ostra del Pacífico (Crassostrea gigas) en la Comuna "La Entrada" de la Provincia de Santa Elena al año 2012. Recuperado de https://dspace.ups.edu.ec/bitstream/123456789/4297/1/UPS-GT000378.pdf

- Wilson, J. 2013. Fundamentals of systems ergonomics/human factors revisited. Applied Ergonomics. 45 (1): 5 - 13.

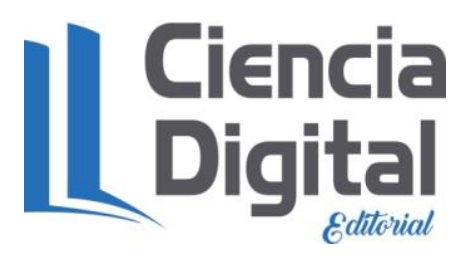




\section{Para citar el artículo indexado.}

Montúfar M., Avendaño U., Ruiz W., Icaza X. \& Alta A. (2018). Prevención de riesgos laborales en la maricultura artesanal de Ecuador. Revista electrónica Ciencia Digital 2(2), 411-427. Recuperado desde:

http://cienciadigital.org/revistacienciadigital2/index.php/CienciaDigital/article/view/106/10 $\underline{0}$

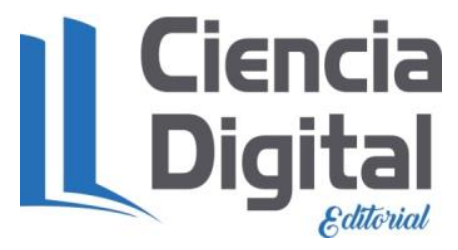

El artículo que se publica es de exclusiva responsabilidad de los autores y no necesariamente reflejan el pensamiento de la Revista Ciencia Digital.

El articulo queda en propiedad de la revista y, por tanto, su publicación parcial y/o total en otro medio tiene que ser autorizado por el director de la Revista Ciencia Digital.
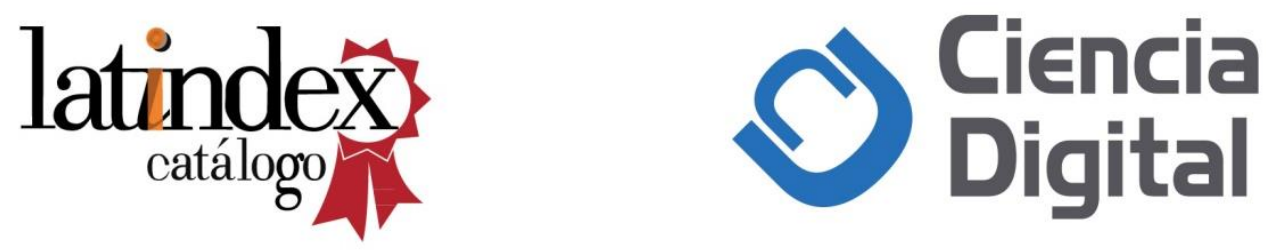